\title{
Educación exitosa para todos: la tutoría como proceso de acompañamiento escolar desde la mirada de la equidad educativa
}

\author{
Successful education for all: tutoring as a process of school support \\ from the perspective of educational equity \\ Brenda Imelda Boroel Cervantes \\ Universidad Autónoma de Baja California, México \\ José Sánchez Santamaría \\ Universidad de Castilla la Mancha, España \\ Karla Denisse Morales Gutiérrez \\ Universidad Autónoma de Baja California, México \\ Patricio Sebastián Henríquez Ritchie \\ Universidad Autónoma de Baja California, México
}

Recibido: 20/09/2018

Aceptado: 18/12/2018

\begin{abstract}
In the Latin American context continues debating the effectiveness in the implementation of educational policies for the development and evaluation of successful educational processes aimed at quality and educational equity. The educational intentions to aspire to a quality education is oriented to strengthen mentoring as a compensatory strategy linked to the process of accompanying school to promote the development of competences, of learning achievement, the taking advantage of opportunities of success for students. The project aimed to describe the perception of young people from high school to the role of the tutor. We used the technique of focus groups a sample cases type formed by students enrolled in the 2018 cycle; the information was analyzed through the inductive analysis of data reduction. Results showed three categories of analysis: a) the teacher tutor roles, b) practices of accompaniment of the teacher tutor as cognitive support and c) approach and trust. The need for training in the non-cognitive dimension (selfcontrol, perseverance and motivation) is evident. Mentoring is recognized as a compensatory strategy that must be implemented to the inside of the school, however, students recognize an incipient atmosphere of trust that prevents the timely follow-up to the needs expressed by the students. It is concluded that programs of mentoring to promote successful education for all as compensatory strategies must link individual student issues with its social and family context.
\end{abstract}

KEY WORDS: Tutoring, compensatory strategies, educational equity, successful education.

\section{RESUMEN}

En el contexto latinoamericano continúa debatiendo la efectividad en la aplicación de políticas educativas para la implementación y evaluación de procesos educativos exitosos orientados a la calidad y equidad educativa. Las intenciones educativas para aspirar a una educación de calidad se orientan a fortalecer la tutoría como estrategia compensatoria vinculada al proceso de acompañamiento escolar para favorecer el desarrollo de competencias, el logro de aprendizajes, el aprovechamiento de oportunidades de éxito para los alumnos. El proyecto tuvo como objetivo describir la percepción de los jóvenes de bachillerato hacia el rol del profesor tutor a partir de las experiencias de los actores involucrados. Se utilizó la técnica de grupos focales a una muestra casos tipo conformada por estudiantes inscritos en el ciclo 2018; la información fue analizada a través del análisis inductivo de reducción de datos. Los resultados arrojaron tres categorías de análisis: a) roles del docente tutor, b) prácticas de acompañamiento del docente tutor como apoyo cognitivo y c) acercamiento y confianza. Se hace evidente la necesidad de capacitación en la dimensión no cognitiva (autocontrol, perseverancia y motivación). La tutoría se reconoce como estrategia compensatoria que debe implementarse al interior de la escuela, sin embargo, los alumnos reconocen una incipiente atmosfera de confianza que impide el seguimiento oportuno a las necesidades expuestas por los alumnos. Se concluye que los programas de tutorías para favorecer la educación exitosa para todos, como estrategias compensatorias deben vincular las problemáticas individuales del alumno con su contexto social y familiar.

PALABRAS CLAVE: Tutoría, equidad, acompañamiento escolar. 


\section{Introducción}

El concepto de calidad educativa vinculada a la educación exitosa puede ser abordado desde varias perspectivas. Diferentes modelos de educación enfocan la calidad desde líneas antagónicas, por un lado, la escuela como el lugar o espacio de lucha política que combate por configurar la calidad como problemática social para así justificar la consecución de políticas educativas determinadas; por otro, problemas objetivos identificados en los procesos formativos, las deficiencias en el aprendizaje de los estudiantes, condiciones vulnerables en los ambientes de aprendizaje y perfiles no idóneos de los profesores en servicio. En ese sentido, el análisis del concepto de calidad ocupa tal presencia en la educación que es difícil de sustituir, sin embargo, no es posible atender el término solo desde los discursos educativos en sus múltiples dimensiones: pedagógicas, sociológicas, psicológicas, históricas y políticas, si primero no se califica como problemática de análisis social. El reto de configurar la calidad educativa como problemática social y con ello, implementar política pública para su solución ha dado origen a la distinción de dos dimensiones altamente vinculadas; la dimensión técnica y la teórica. La primera define las categorías a ser incluidas como parte de la problemática y los componentes para su seguimiento, evaluación y toma de decisiones de acuerdo al resultado. Como parte de tal dimensión se diseñan, clasifican y jerarquizan los diferentes indicadores como eficiencia, eficacia, pertinencia, relevancia y equidad a fin de determinar la cercanía o lejanía de lo que se ha determinado como bueno. La segunda ubica a la calidad como problema teórico, la cual coloca a la educación como elemento constitutivo de la justicia social; la calidad desde este enfoque establece niveles de logro no para producir desigualdad, sino para identificar la existente al interior del sistema educativo (Plá, 2018).

En este sentido, una de las crecientes preocupaciones políticas y sociales actuales en muchos países en torno a la educación reside en promover la puesta en marcha de procesos educativos para todos, es decir, impulsar una educación exitosa a partir de procesos de acompañamiento que aspiren a ser de calidad. Los países en Latinoamérica continúan enfrentando enromes desafíos en materia de procesos educativos exitosos orientados a la calidad y equidad educativa. Las directrices en política pública han orientado su atención principalmente en el incremento de acceso y cobertura; sin embargo, las opciones educativas para dar respuesta a las necesidades socioculturales y escolares de la creciente población estudiantil no son suficientes para garantizar su permanencia y conclusión educativa exitosa. El presente proyecto aborda como eje el movimiento de educación exitosa para todos, desde los procesos de acompañamiento, mismo que se centra en proveer una educación de calidad reconociendo las diferencias de cada estudiante. Esta realidad obliga a pensar las políticas de equidad e inclusión desde una perspectiva distinta. En este sentido, la UNESCO (2015), cuestiona la eficacia de las aspiraciones de las políticas educativas modernas por avanzar hacia sociedades más justas, cohesivas y democráticas; la búsqueda asociada a generar bienestar social y calidad de vida para todos; una educación primaria universal; ampliación y mejora de la atención y educación de la primera infancia; la reducción del analfabetismo; la igualdad de acceso al aprendizaje para jóvenes y adultos; eliminación de la disparidad entre géneros o la mejora de la calidad de la educación con resultados mesurables.

La evidencia empírica disponible nos informa que la calidad educativa actúa como marco de referencia del rol del docente que aspire a promover el éxito educativo de todos sus estudiantes, en torno a la cual la equidad es un aspecto esencial, entendida como un principio pedagógico que articulando la inclusión y la justicia social, se caracteriza por generar un estado del proceso educativo orientado al éxito educativo para todos (Sánchez-Santamaría y Manzanares-Moya, 2017). En este sentido, se pretende, en última instancia generar un conocimiento, relacionado con el rol del docente tutor en la implementación del programa de tutorías como estrategia y la construcción de relaciones de confianza entre tutores y alumnos a fin de mejorar la educación exitosa de calidad en la Educación Media Superior en la Baja California desde una perspectiva de equidad y justicia social. En el ámbito de la tutoría en educación media superior, las aportaciones son aun incipientes, la literatura muestra reportes empíricos que denotan resultados cuantitativos que aportan indicadores para la evaluación de la operación del programa de tutorías y del servicio que ofrecen los tutores (Barcelata- Eguiarte, Gómez- Gutiérrez y Taboada 2010; Ducoing, 2009; Muñoz, 2004 y Robles 2005), sin embargo, la 
línea de investigación en torno a las experiencias cualitativas durante el proceso de acompañamiento, que experimentan los actores involucrados, se encuentra en fase de desarrollo (Trujillo, 2013).

\section{Equidad, inclusión y justicia social: contexto.}

Las opciones generadas dentro y fuera de las escuelas para dar respuesta a las necesidades socioculturales y escolares de la creciente población estudiantil no son suficientes para garantizar su permanencia y conclusión educativa exitosa. Si bien, la educación es un bien público que los Estados ponen al servicio de la ciudadanía porque tiene un relevante papel en la formación y actualización de la misma para hacer frente a los retos políticos, sociales y económicos de la sociedad del conocimiento, debe alcanzar simultáneamente los objetivos de equidad, pertinencia y calidad (UNESCO, 2009; OCDE, 2015). Esto se traduce en una mejora del bienestar del conjunto de la sociedad.

Esta realidad obliga a pensar las políticas de equidad e inclusión desde una perspectiva distinta. La evidencia empírica disponible nos informa de que la calidad educativa vinculada a la equidad es un aspecto esencial, entendida la equidad como "un principio pedagógico que articulando la inclusión y la justicia social, se caracteriza por generar un estado del proceso educativo orientado al éxito educativo para todos" (Sánchez-Santamaría y Manzanares-Moya, 2017). Las aportaciones teóricas en la definición de la equidad educativa no están acabadas. La UNESCO (2002), por ejemplo, la refiere como tomar en cuenta la desigual situación de los alumnos y sus familiares, de las comunidades y escuelas, ofrecer apoyos especiales a quienes lo requieran, de tal forma que los objetivos sean alcanzados por el mayor número de estudiantes; su construcción sostiene dos elementos, inclusión y justicia; por otro lado, Sánchez y Manzanares (2013), la retoman como elemento constitutivo de valor social de la educación social que se articula en torno a un conjunto de discursos y prácticas educativas orientadas a promocionar y mejorar el desarrollo competencial de todos los estudiantes de acuerdo a tres dimensiones: inclusión, justicia y diversidad cohesiva. Muñoz Izquierdo (2009), precisa que la equidad se trascribe en "las oportunidades de recibir educación y de participar de los beneficios sociales derivados de ella deben distribuirse equitativamente entre los diversos sectores de la sociedad" (p.29). En este sentido, Silva-Laya (2011), aporta que la equidad en educación superior está fundamentada en un principio de justicia social que contempla tres condiciones, el acceso, la compensación de desigualdades, permanencia y la obtención de resultados afirmativos y significativos. Si bien, las definiciones contienen elementos distintivos y definitorios desde una visión integral, llama la atención la falta de contextualización en el marco de una sociedad desigual y, con ello, queda ausente la necesidad de compensar las desventajas, condición que juega un rol determinante en el acceso y permanencia efectiva en la educación superior (Flores Crespo, 2009).

Sabemos que, en general, "los sistemas educativos con mayores niveles de rendimiento académico de los países de la OCDE son aquellos que combinan una elevada calidad y equidad" (OCDE, 2012, p. 15). Esto implica asumir el principio de equidad en interacción con el de calidad para garantizar la igualdad de oportunidades en el acceso, la participación y resultados en la Educación, de modo que no medien variables políticas, culturales, económicas y/o familiares. Lo que se traduce en diseñar políticas, programas y prácticas que reconozcan la diversidad como un valor, de modo que se promueva la inclusión y la participación en la educación desde la justicia social: el acceso a la educación, la eliminación de barreras que impidan la participación y la calidad vinculada con el rendimiento académico y el impacto social, así como los elementos asociados a la permanencia y promoción a lo largo de la vida académica del estudiante en la escuela.

\section{Educación para todos: retos del acompañamiento escolar.}

En Baja California, el punto de partida en la protección de la equidad se establece en su Constitución Política, la cual instituye que toda persona tiene el derecho a gozar de un medio ambiente adecuado para su desarrollo y bienestar, así mismo, el derecho a la igualdad y a la no discriminación para desarrollar armónicamente todas las facultades del ser humano y fomentar en él, 
el amor a la Patria, el respeto a los derechos humanos y a la conciencia de la solidaridad internacional, en la independencia y en la justicia. Aunado a ello, el Plan Estatal de Desarrollo 2014 - 2018, presenta como objetivo asegurar la calidad de la formación profesional en la Educación Media Superior, a través de programas y proyectos institucionales acordes a las necesidades que requiere el desarrollo social y económico de Baja California. Como iniciativas propone incrementar la capacidad y diversidad de opciones de acceso y permanencia asegurar la calidad de la oferta educativa y mejorar la práctica y el desempeño docente en apego a los nuevos modelos educativos propuestos (p. 281). Sin embargo, en el Estado, de acuerdo a los indicadores de las cifras de absorción de matrícula van en decremento, mientras que en el ciclo escolar 2013-2014, ingresaron, un total de 446 alumnos, de los cuales en el ciclo escolar 2015-2016 solo egresaron 294 alumnos. Lo que representa un 34.1\% de deserción y abandono escolar. Los esfuerzos del Sistema Estatal de Educación media superior para atender y retener la demanda educativa y el crecimiento de la matrícula han disminuido. Esto significaría que el esfuerzo público para atender las presiones demográficas no ha sido suficiente. En la actualidad, no se cuenta con una política educativa que equilibre los intereses federales con los estatales, tomando poco en cuenta el desarrollo económico, político y social diferenciado además de la carencia de evidencia empírica que describa los mecanismos de evaluación y control administrativo, a partir de los criterios de equidad de acceso, calidad y pertinencia.

El reto en la Educación Media Superior (EMS) consiste en brindar formación integral a los estudiantes para la adquisición de habilidades que les permita enfrentar problemas relacionados con las demandas del mundo moderno. Alcanzar dicho fin, representa un desafío complejo para la Secretaria de Educación Pública (SEP), debido a la su obligatoriedad, establecida en la Ley General de Educación (LGE, 2013), acceden al sistema alumnos provenientes de segmentos de la población antes no considerados, alumnos con diversidades socioeconómicas, socioculturales y cognitivas. Es decir, las aulas que ocupan actualmente nuestros estudiantes están conformadas de manera heterogénea, motivo por el cual, el establecer relaciones de cercanía, en los aspectos; fiscos, intelectuales y afectivos así como confianza y respeto reciproco entre docente tutores y estudiantes resulta de suma importancia en pro de perfeccionar del proceso formativo integral del estudiante (González, 2008).

De lo anterior, la importancia de brindar al estudiante un acompañamiento genuino, que considere sus particularidades, y que las mismas sean reconocidas y valoradas, como característica inherente del ser humano Amaro, Méndez y Mendoza, (2014), al respecto definen:

La necesidad de atender a la diversidad de una forma integral, es decir que las instituciones fomenten una educación que responda a las características de todos los estudiantes, que los ayude a sentirse participes y conciba sus diferencias como un recurso de aprendizaje común (pp.201).

Las carencias de acompañamiento, provocan que el alumno no se sienta parte de su contexto, lo que sin duda contribuye a que su interés por continuar sus estudios disminuya o en el peor de los casos sientan rechazo, tal como lo identificado por Ortiz, Mendoza y Méndez (2016). Al respecto, un estudio realizado por Ortiz, Mendoza y Méndez (2016), cuyo objetivo consistió en comprender lo procesos socioeducativos que llevaron a los estudiantes a abandonar sus estudios de bachillerato, dentro de los principales resultados destacan: la falta de acompañamiento por parte de sus profesores tutores así como actitud excluyente del profesor hacia algunos de los alumnos. Ante lo que se concluye en el mismo, que la falta de reconocimiento de habilidades de los estudiantes así como dejar de lado sus interese y opiniones personales, puede provocar actitudes de rechazo por parte de los estudiantes ente el estudio.

\section{La diversidad del alumnado en la educación media superior}

Los jóvenes enfrentan problemas relacionados con las exigencias del mundo globalizado, el impacto de dichas problemáticas se materializan en el ingreso y permanencia en la escuela. Bajo estas 
situaciones su proyecto de vida depende principalmente de las oportunidades de formación educativa y sobre todo de superar las carencias asociadas a su entorno social. En ese sentido, las Instituciones de Educación Media Superior deben redoblar sus esfuerzos en la implementación de mecanismos para eliminar los obstáculos que puedan truncar los alumnos en su proceso formativo. Sin embargo, al interior de los centros escolares, se parte del hecho de que las aulas estén conformadas de manera heterogénea, conlleva que el docente reconozca al estudiante como poseedor de cualidades únicas, y distintos estilos y ritmos de aprendizaje, por lo que se considera prioritaria la utilización de distintas estrategias mediante las cuales sea posible prestar atención a la diversidad (Ortiz, Mendoza y Méndez, 2016).

Cabe señalar que la diversidad, no representa a las asimetrías existentes entre las personas o grupos, sino que son inherentes a la naturaleza humana, Blanco (s.f) y en palabras del autor "son utilizadas para segregar, seleccionar discriminar a los estudiantes" (p.92). Aunado a ello, conforme los alumnos avanzan en su trayectoria académica, las instituciones educativas pierden de manera paulatina la capacidad para implementar estrategias compensatorias que aseguren su permanencia, lo cual limita de manera indirecta las proyecciones de vida y carrera de la comunidad estudiantil, con ello, pierden oportunidades de apoyo para perfeccionar nuevas competencias al interior del contexto educativo (Landero, 2012).

Entre las diferencias existentes en el alumnado se encuentran las identificadas como por Silva (2015), como diferencias de inicio, refiere a las diferencias existentes respecto al bagaje de conocimientos adquiridos por los estudiantes, al no ser identificadas y atendidas, se convierten en factores que ponen en riesgo la permanencia y egreso de los estudiantes.

En este tenor, México ha implementado acciones compensatorias para abatir problemáticas de rezago y abandono escolar; se identifica entre las políticas educativas, el decreto publicado en el Diario Oficial de la Federación (DOF), publicado en marzo de 2012, en el cual se fortalece al Consejo Nacional de Fomento Educativo (CONAFE) como instancia que promueve equidad e igualdad en la educación inicial y básica en las localidades rurales con mayor rezago educativo. CONAFE, fue creado con el objeto de allegarse recursos complementarios, económicos y técnicos, nacionales o extranjeros para aplicarlos al mejor desarrollo de la educación en el país (DOF, 2016). En ello, se declaran programas compensatorios que buscan reducir el rezago educativo mediante el apoyo de recursos específicos a los estados de la República, previa celebración de convenios en los que se concierten las proporciones de financiamiento y las acciones específicas que las autoridades educativas locales deban realizar para reducir y superar dichos rezagos.

Entendemos el término compensación como equiparar, re-equilibrar, igualar en sentido opuesto, dar alguna cosa u otorgar algún beneficio en resarcimiento del daño que se ha causado. En el ámbito educativo Muñoz, et al, (1992), (citado en Sánchez y Manzanares, 2017), aluden a las estrategias compensatorias considerándose como aquellas que tienen como objeto contribuir a solucionar los problemas de desigualdad en las que se encuentran ciertas personas como consecuencia de determinados factores económicos, sociales y/o geográficos.

Las estrategias compensatorias se orientan a fortalecer el desarrollo de habilidades cognitivas y no cognitivas en los estudiantes. Por cognición se entiende la capacidad que tienen los seres vivos de procesar la información a partir de la percepción, el conocimiento adquirido con la experiencia y nuestras características subjetivas que permiten integrar toda esta información para valorar e interpretar el mundo. Es decir, la cognición es la habilidad que tenemos para asimilar y procesar los datos que nos llegan de diferentes vías para convertirlos en conocimiento. Se estructura de diferentes procesos cognitivos como el aprendizaje, la atención, la memoria, el lenguaje, el razonamiento, la toma de decisiones, etcétera, que forman parte del desarrollo intelectual y de la experiencia. Las habilidades no cognitivas en tanto son aquellas que proveen las bases y el soporte para el desarrollo de las habilidades cognitivas, al agrupar rasgos de personalidad y actitudes que facilitan el aprendizaje y permiten el desarrollo del conocimiento. En este sentido, se hace referencia a las habilidades no cognitivas como aquellas independientes de capacidad intelectual, entre dichas habilidades se recupera el autocontrol, la perseverancia y la motivación como los atributos y capacidades del ser humano. Labrador, Cruzado y Muñoz 1993, (citado en Luna, Gómez y Lazo 2012), definen el 
autocontrol como la función de disminuir la probabilidad de ocurrencia de una conducía o conjunto de las mismas, que en algún momento podrían resultar aversivas para la persona que se comporta (p.52), se entiende también como la capacidad consciente de regular nuestros impulsos de manera voluntaria, a fin de alcanzar un mayor equilibrio personal y relacional. La perseverancia es la fuerza de voluntad que nos lleva a concluir algo que nos propusimos hacer, aunque surjan dificultades internas o externas, o a pesar de que disminuya la motivación personal. Es la predisposición a mantenerse firme y constante en la prosecución de acciones y emprendimientos de manera estable o continua hasta lograr el objetivo. Por su parte Núñez (2009), considera a la motivación como un conjunto de procesos implicados en la activación, dirección y persistencia de la conducta. Alcalay y Antonijevic 1987 (citado en Navarro, 2003) contribuye a la conceptualización de motivación al referirse a la motivación escolar como el proceso involucra variables tanto cognitivas como afectivas: cognitivas en cuanto a habilidades del pensamiento y conductas instrumentales para alcanzar las metas propuestas; afectivas en tanto comprende elementos como la autoevaluación, auto concepto, etc. Se asume que cuando se disfruta ejecutando una tarea se induce una motivación intrínseca positiva. La motivación que procede de fuera y que conduce a la ejecución de la tarea.

\section{La tutoría como proceso de acompañamiento del estudiante}

Con la intención de prestar atención a las particularidades de los estudiantes en la EMS, resulta de vital importancia, que este sea acompañado durante su proceso con la intención de brindar atención oportuna en caso de presentar alguna situación problema de índole particular que ponga en riesgo su formación.

El acompañamiento, es conceptualizado por Puerta Gil (2016) como:

acción educativa intencionada que se fundamenta en la cercanía, en la disposición para afectar y dejarse afectar por el otro, teniendo como horizonte primordial la potencialización de sus capacidades, de tal manera que este siga configurando y moldeando sus sueños y esperanzas (s.f.).

En este contexto y con la intención de enfrentar los retos de la EMS, la Subsecretaría de Educación Media Superior (SEMS), establece el Programa "Síguele, caminemos juntos", Acompañamiento Integral para los Jóvenes de la Educación Media Superior (SEP, 2011), con la intención de articular acciones que favorezcan la formación integral de los estudiantes, así como la prevención de la deserción, incrementar el aprovechamiento académico y la eficiencia terminal, para lo cual se plantea el siguiente objetivo:

mejorar el aprovechamiento escolar de los estudiantes, ofreciendo un adecuado desarrollo de conocimientos, habilidades y actitudes que les permitan una acertada toma de decisiones para su proyecto de vida, profesional y laboral, además de contribuir a incrementar la eficiencia terminal, y disminuir los índices de deserción y reprobación (p.12).

Dicho programa se estructura a partir de seis dimensiones: 1) (SIAT) Sistema de Alerta Temprana (generadora de acciones oportunas); 2) (SINaTA) Sistema Nacional de Tutorías Académicas (atiende el aspecto académico); 3) Programa de Orientación Vocacional (se orienta al área vocacional); 4) Construye T (se enfoca al ámbito psicosocial y afectivo); 5) Programa de Becas (dimensión socioeconómica) y 6) Programa de Fomento a la Lectura.

La actividad tutorial, mediante la cual se brinda atención a los estudiantes se encuentra dividido en tres instancias: Sistema Nacional de Tutorías Académicas (SiNaTA), el programa de Orientación Vocacional y Construye $\mathrm{T}$.

Cabe resaltar que si bien la acción tutorial está ligada a contribuir con la disminución de la deserción y reprobación en este nivel educativo, es también es el espacio ideal para brindar atención 
y apoyo a los jóvenes que durante su proceso de formación enfrentan situaciones problemas, ante las cuales deben tomar decisiones que traen consigo consecuencias para toda la vida. (Acuerdo 9 SNB). La SEP (2017) conceptualiza a la tutoría como:

Aquel proceso de acompañamiento mediante una serie de actividades organizadas que guían al estudiante para recibir atención educativa personalizada e individualizada de parte del docente a cargo, por medio de la estructuración de objetivos, programas, organización por áreas técnicas de enseñanza apropiadas e integración de grupos conforme a ciertos criterios y mecanismos de monitoreo y control, para ofrecer una educación compensatoria o enmendadora a las alumnas y los alumnos que afrontan dificultades académicas (p.127).

Con la propuesta de Nuevo Modelo Educativo en el año de 2016, el cual encuentra sustento en lo establecido en la Reforma Integral de Educación Media Superior (RIEMS), con base al acuerdo 442 , donde se establece como eje principal: La construcción del Marco Curricular Común (MCC) en base a competencias.

Bajo este contexto, la figura del docente tutor toma relevancia, (ACUERDO número 9/CD/2009 del Comité Directivo del Sistema Nacional de Bachillerato) adquiriendo un lugar preponderante en el quehacer escolar. Entre las funciones de este actor escolar se encuentra el "facilitar el desarrollo personal de los estudiantes en el marco de sus necesidades e posibilidades como individuos teniendo en cuenta las circunstancias que los rodean" (p.2).

Con base a lo antes expuesto, resulta necesaria la existencia de lazos de confianza y cercanía que permita al docente tutor identificar los aspectos relevantes de los estudiantes, debido a que las competencias cognitivas se ponen en juego si el alumno no desarrolla habilidades socioemocionales (SEP, 2018).

\section{Proceso metodológico}

Se realizó un estudio cualitativo, en concreto, se empleó el diseño fenomenológico, cuyo objetivo consistió en comprender desde la perspectiva de los estudiantes cómo se construyen las relaciones de confianza entre los estudiantes y sus docentes tutores en un centro escolar de Educación Media Superior, en el noroeste de México.

Lo anterior, con base en a lo propuesto por Colmenares y Piñero (2008), quienes señalan que a través de esta perspectiva es posible tomar decisiones a partir de las experiencias y vivencias compartidas por quienes se encuentran en los lugares en donde se circunscriben los hechos.

La muestra se confirmó por quince estudiantes distribuidos en tres grupos, dos de seis y uno de cinco respectivamente, integrados por estudiantes matriculados a la institución, de los cuales diez eran hombres y siete mujeres, la selección responde al muestreo casos tipo, como criterio de selección, estudiantes de diferente grado escolar y, que hayan vivido la experiencia de recibir tutoría al menos en una ocasión (ver Tabla 1).

\subsection{Técnica para la recolección de datos}

Los datos observados se obtuvieron mediante la aplicación de la técnica grupo focal a estudiantes, por posibilitar obtener información desde actores involucrados. McKernan (2001), define la técnica como aquella que "pretende proporcionar materiales para el desarrollo del juicio práctico de los actores en situaciones problemáticas" (p. 24). 
Tabla 1.

Descripción de la muestra de estudio por grupo focal

\begin{tabular}{|c|c|c|}
\hline \multicolumn{3}{|c|}{ Grupo Focal 1 } \\
\hline Género & Grado / semestre & Edad \\
\hline 3 hombres y 3 mujeres & 1ro y 2do semestre & $\pm 16 \pm 18$ \\
\hline \multicolumn{3}{|c|}{ Grupo Focal 2 } \\
\hline Género & Grado / semestre & Edad \\
\hline 4 hombres y 1 mujer & 3er y 4to semestre & Edad \\
\hline \multicolumn{2}{|c|}{ Grupo Focal 3 } \\
\hline Género & Grado / semestre & $\pm 18 \pm 19$ \\
\hline 3 hombres y 3 mujeres & 5to y 6to semestre &
\end{tabular}

\subsection{Procedimiento}

En la presente investigación se llevó a cabo la aplicación de la técnica grupos focales, dentro de las actividades realizadas, se encuentran las siguientes: Posterior a la autorización de acceso al centro escolar, se acordó de manera personal con los estudiantes la hora y el espacio para la realización de la técnica. Se les explicó a los alumnos el objetivo del estudio y se les solicitó su participación voluntaria el día y hora programada. De la misma manera, se les hizo saber que la información observada durante la sesión, seria audio grabada con la intención de rescatar información relevante para el objetivo de la investigación, y tratada bajo el criterio de confiabilidad, por lo que no tendría repercusión alguna en su trayectoria educativa. Una vez que los estudiantes aceptaron de forma voluntaria participar en el estudio, se realizó el grupo focal, este procedimiento se aplicó en cada una de las sesiones. La técnica de grupos focales, permite la recogida de información de gran utilidad para acceder a un nivel de comprensión profundo acerca de las opiniones, percepciones, valoraciones, etc., de una audiencia relevante sobre las prácticas y condiciones de desarrollo de la investigación. De este modo, con esta técnica se establece un clima de discusión que permite conocer los significados que, los actores intervinientes en el mismo, otorgan al desarrollo del tema estudiado. Se trata de un proceso de construcción discursiva caracterizado por un ejercicio de descripción sobre las posiciones adoptadas por los actores, bien a partir de un análisis lógico (descriptivo) o de un análisis interpretativo (comprensivo).

La conformación del grupo focal fue: a) un moderador, responsable del proyecto de investigación, además de guiar la conversación entre los participantes y, b) estudiantes de los tres grados escolares, con el objetivo de obtener su información de estudiantes de recién ingreso, de etapa intermedia y potenciales a egresar del bachillerato. El grupo focal se desarrolló en las instalaciones de la institución, se buscó generar un clima de intimidad y comodidad entre los participantes, fue audio grabada bajo el consentimiento de los participantes.

\subsection{Análisis de la información}

La transcripción de los grupos focales fue textual, se consideró la totalidad del audio del grupo. En lo referente a la técnica de reducción de datos, se establecieron categorías y unidades de análisis acordes al objetivo del proyecto y los datos obtenidos. El procedimiento de análisis siguió la propuesta de (Titscher, Meyer, Wodak y Vetter 2000). Las unidades de análisis fueron codificadas e incorporadas a una categoría correspondiente. En este sentido, las unidades de análisis fueron unidades gramaticales, dado que se tomaron las oraciones que enfatizan los aspectos formativos referentes a los principios y valores ético profesionales. Se obtuvieron inicialmente siete categorías, mismas que fueron reducidas a tres en un segundo análisis. Se utilizó análisis de contenido de tipo inductivo (Hernández y Mendoza 2018), con el objetivo de asociar códigos con fragmentos del texto, y crear proyectos denominados "unidades hermenéuticas" (UH) que incluyen documentos primarios como por ejemplo, citas, códigos y memos. 
Las categorías que responden al interés del estudio fueron las siguientes: a) Funciones del docente-tutor, b) Prácticas de acompañamiento del docente-tutor como apoyo cognitivo y c) Acercamiento y confianza.

\section{Resultados}

En este apartado se presentan los resultados obtenidos, la ruta de análisis que brindó hallazgos de mayor triangulación y sentido fue a partir de la descripción de cada una de las categorías de análisis, construidas a partir de los grupos focales realizados a los jóvenes. Así mismo se presentan ejemplos de algunos códigos utilizados y fragmentos de unidades de análisis.

Los resultados se encuentran organizados en tres categorías, las cuales se definen a continuación:

\subsection{Funciones del docente tutor}

Esta categoría hace referencia a la implementación de acciones educativas y sociales que los docentes desde su rol de tutores desempeñan de manera continua para el seguimiento de la trayectoria personal y académica de los estudiantes en su paso por el bachillerato.

En que referente a la primera categoría, se encontró que los alumnos identifican principalmente las funciones del docente-tutor como aquella figura a la que pueden acudir si enfrentan alguna dificultad académica, es identificado por uno de los participantes del a siguiente manera:

"Él nos ayuda a bacer los trabajos que necesitamos para materias que hayamos reprobado, problemas con maestros, asesorias y asi”".

Algunos de los fragmentos más significativos se muestran en la Tabla 2, agrupados en la categoría Funciones del Docente Tutor [FDT].

Tabla 2.

Funciones del docente tutor en el proceso de acompañamiento escolar

\begin{tabular}{|l|l|}
\hline \multicolumn{1}{|c|}{ Categoría } & \multicolumn{1}{c|}{ Unidades de análisis } \\
\hline Funciones del docente & P4 gfl "Él nos ayuda a hacer los trabajos que necesitamos para materias \\
que hayamos reprobado". & P3 gf2 "Por ejemplo, si tienes un problema o algo así, él te pueda explicar \\
y eso, o con un profesor, si tienes problemas" & P1 gf2"Problemas con maestros, asesorías". \\
& P2 gf3 "Pues es que no pregunta por la materias que vamos a reprobar y \\
& no ayuda para pasarlas, no manda con el profesor a las asesorías" \\
\hline
\end{tabular}

Fuente: Elaboración propia.

De lo anterior, se asume que el docente-tutor realiza prácticas en pro de apoyo académico de los alumnos tal como lo establece el manual de tutorías de la Subsecretaria de Educación Media Superior a través de los Lineamientos Generales de la Tutoría en el Sistema Nacional de Bachillerato (2009), aquí mismo se identifica entre las habilidades con las que debe contar el docente tutor está la habilidad de identificar problemas académicos, psicosociales y afectivos de los estudiantes. Sin embargo, en lo que respecta al apoyo socioemocional, dicha función no es identificada por los alumnos. 
7.2. Prácticas de acompañamiento del docente tutor como apoyo cognitivo

La tutoría entiende el proceso de acompañamiento no solo en el aspecto académico del estudiante, sino en su desarrollo y crecimiento personal, en esta categoría se describen particularmente, los mecanismos y estrategias de las que hace uso el profesor - tutor para atender las necesidades cognitivas y de aprendizaje de los alumnos, consideradas esenciales en el proceso de tutoría, su utilidad radica en reconocer sus habilidades de organización del tiempo, hábitos de estudio y estrategias de aprendizaje.

En esta categoría los jóvenes identificaron la figura del docente-tutor como ajena a su entorno, puesto que los alumnos que durante su formación han presentado algún problema de índole personal, no han tenido confianza con su docente-tutor para solicitar apoyo y aquellos que no han experimentado alguna problemática refieren que en caso de tenerla no tendrían confianza para solicitar apoyo. Es decir; no existen los lazas de confianza necesario para mediar entre el alumno el medio. Al respecto, uno de los participantes refirió lo siguiente, cuando se le pregunto si acudiría a su docente-tutor en caso de presentar una situación problema de índole personal o familiar:

"no tendría la confianza de platicarle mis cosas a una persona que no conozco",

Los fragmentos de las unidades de análisis arrojadas del proceso de reducción de datos se agruparon en la categoría Prácticas de Acompañamiento del Docente-Tutor como apoyo Cognitivo [PDTAC], se presentan en la Tabla 3.

Tabla 3.

Prácticas de acompañamiento del docente tutor como apoyo cognitivo.

\begin{tabular}{|l|l|}
\hline \multicolumn{1}{|c|}{ Categoría } & \multicolumn{1}{c|}{ Unidades de análisis } \\
\hline $\begin{array}{l}\text { Prácticas de } \\
\text { acompañamiento del } \\
\text { docente-tutor, apoyo } \\
\text { cognitivo [PDTAC] }\end{array}$ & $\begin{array}{l}\text { P1gf "Les hablan a nuestros papás si vamos reprobando materias y ya ven } \\
\text { pues cómo le hacen, que problemas tenemos, y en qué materias, los } \\
\text { trabajos, las faltas sobre todo". } \\
\text { P2gf "Por ejemplo, nosotros que ya vamos a salir, nos ayudan para ver de } \\
\text { lo que queremos ser, qué carrera elegir y todo eso". } \\
\text { P3gf3 "Pues él nos ayuda preguntándonos que es lo que no entendemos... } \\
\text { y nos dice que podemos preguntarle al profe de esa materia, cómo le } \\
\text { podemos hacer, para pues, no reprobar la materia" }\end{array}$ \\
\hline
\end{tabular}

Fuente: Elaboración propia.

Una relación de confianza propicia un clima apto para el dialogo, así como para la resolución de conflictos y problemas (Conejeros, Rojas y Segura, 2010). Este aspecto, es identificado en los Lineamientos Generales de la Tutoría en el Sistema Nacional de Bachillerato (2009) como parte del perfil de los actores de la acción tutorial, esto con la intención de que el alumno se sienta en la libertad de expresar sus ideas e inquietudes. Con base a lo antes mencionado, se concluye en la institución receptora no existen los lazos de confianza necesarios para mediar entre el alumno y el medio.

\subsection{Acercamiento y confianza}

En esta dimensión se describe la percepción de los jóvenes hacia el interés y disposición personal que muestra el docente-tutor en el establecimiento de ambientes favorables para la acción tutorial, son considerados como elementos importantes para su permanencia y el logro de un buen desarrollo personal y académico en el bachillerato.

En lo que respecta a la tercera categoría, se identificó que los alumnos no perciben al docente -tutor interesado en sus aspectos personales, uno de los estudiantes expresó lo siguiente: 
"los maestros no se acercan de esa manera a nosotros, simplemente ellos aplican el programa de trabajo que les dan, tú te tienes que acercar a ellos, ellos no se acercan a ti”.

La Tabla 4 señala los discursos de los jóvenes que participaron en el estudio en relación a la categoría Acompañamiento y Confianza con el Docente -Tutor [ACDT].

Tabla 4.

Acercamiento y confianza con el docente tutor.

\begin{tabular}{|c|c|}
\hline Categoría & Unidades de análisis \\
\hline $\begin{array}{l}\text { Acercamiento y confianza } \\
\text { con el tutor }[\mathrm{ACDT}] .\end{array}$ & $\begin{array}{l}\text { P2gf1 "Pues, es que uno no le platica sus cosas a cualquier persona". } \\
\text { P4gf2 "Como es también mí maestro en otra clase, pues como te vas a } \\
\text { poner a hablar tus cosas personales". } \\
\text { P2gf3 "Es que como es maestro en otra materia, no se sabe cómo lo } \\
\text { pueda tomar". } \\
\text { P3 gf4 "no, porque no tendría la confianza de platicarle mis cosas a una } \\
\text { persona que no conozco". } \\
\text { P5 gf4 "Los maestros no se acercan de esa manera a nosotros, } \\
\text { simplemente ellos aplican el programa de trabajo que les dan, tú te } \\
\text { tienes que acercar a ellos, ellos no se acercan a ti" }\end{array}$ \\
\hline
\end{tabular}

Fuente: Elaboración propia.

En un estudio regional realizado por Osuna y Díaz (2015), se encontró que más del 70 \% de los alumnos indicó que a sus profesores no les interesaba conocer sus aspectos familiares o de su vida personal. Este aspecto, es señalado en los lineamientos Generales de la Tutoría en el Sistema Nacional de Bachillerato (2009), aquí se identifica como parte del perfil del docente-tutor el interés por situaciones no sólo académica de los estudiantes, es decir prestar atención las cuestiones personales que, personales, la escucha, la empatía y comprensión para con el alumnado. Con base a lo antes mencionado se concluye que se percibe por parte de los alumnos participantes falta de interés del docente-tutor por sus aspectos personales.

\section{Conclusiones}

Para erradicar la desigualdad en los contextos educativos en México, se ha apostado a la implementación de políticas compensatorias sobre la base del acceso a la educación obligatoria, básica y media superior. Sin embargo, los esfuerzos no han sido suficientes para garantizar la permanencia y el desarrollo de facultades o competencias adecuadas que nos acerquen a la dimensión instrumental de la calidad. Si recurrimos al modelo clásico de abandono escolar propuesto por Tinto 1975 (citado en García y Androgué, 2015), se debe privilegiar desde la investigación educativa el estudio entre la interacción entre el individuo y la institución para explicar el proceso de toma de decisiones de los estudiantes para perder su identidad como estudiante y eventualmente abandonar su educación formal. Como respuesta a ello, las estrategias compensatorias que deben implementarse al interior de la escuela, mediante apoyos a programas que garanticen el acompañamiento para desarrollo de competencias, el logro de aprendizajes, el ofrecimiento de oportunidades de éxito para los alumnos. En este tenor, los profesores deben contar con capacitación pertinente para atender y resolver las diferencias individuales para potencializar las capacidades de los alumnos y propiciar la construcción de una cultura común e igualitaria. Como país, particularmente, en el ámbito educativo nos hace falta 
transitar del modelo clásico de equidad entendida como distribución social de provisiones, ingresos, recursos, derechos basados en la justicia social y distributiva hacia el modelo de equidad contemporáneo, que implica ubicar la equidad como igualdad de oportunidades y eliminación de barreras económicas, sociales y políticas que impiden el desarrollo del alumno. Los procesos educativos y de acompañamiento escolar deben generar experiencias y situaciones exitosas para todos. Para ello se deben conjugar la equidad a través de la diversidad y la equidad a través de la ciudadanía; para que la equidad educativa sea efectiva y extensiva, es preciso dotar de un valor social que favorezca la construcción de prácticas educativas de éxito para todos. Se tiene conocimiento que la escuela no lo puede resolver todo, pero si tiene entre sus funciones fundamentales el formar ciudadanos comprometidos con los valores democráticos en el sentido de que la sociedad se construye desde el interior de los centros escolares, y no al margen de ella (Sánchez-Santamaría, 2011).

En la presente investigación, los resultados muestran que en el proceso de tutoría como programa institucional de acompañamiento de los alumnos del bachillerato, se involucran diversos aspectos con el contexto personal (socio afectivo) y académico (de aprendizaje). Asimismo, se presentan elementos relacionados con la práctica del docente tutor al interior del centro escolar así como la construcción social de la problemática en la institución de la incipiente capacitación del docente tutor para atender aspectos socio afectivos y necesidades individuales en la comunidad de estudiantes. Esto se concluye debido a que los estudiantes manifiestan dificultades para establecer relaciones de confianza y de comunicación asertiva. De igual manera, algunas actitudes de los profesores en clase pueden dejar en evidencia sus intereses y opiniones personales manifestados en las sesiones de tutoría. Por tanto es importante que el docente tutor considere que cada alumno tiene cualidades únicas y ritmos de integración al centro escolar diferentes, por lo que dentro de sus funciones está la detección de necesidades académicas y personales y así diversificar los mecanismos y estrategias de tutoría desde una mirada de equidad de proceso, así como motivarlos y acompañarlos a lo largo de su formación académica. Para cumplir además con el propósito de favorecer la formación de la ciudadanía del futuro coadyuvando a que los jóvenes adquieran destrezas, habilidades, capacidades que le permitan saber, saber hacer, ser y convivir en sociedad (Moreno-Crespo, 2015).

Resulta necesario, continuar con investigaciones a partir de estudios diagnósticos in situ, que cedan la voz a los estudiantes y a través de estas, indagar en los aspectos subjetivos de las tutorías, con la intención de identificar posibles áreas de intervención en pro de mejorar los procesos socio afectivos que posibiliten relaciones de cercanía y confianza entre maestros tutores y sus estudiantes. Bajo este contexto, Gómez y Collado (2012), han aportado de manera significativa en el tema al realizar un estudio cuya intención radicó en conocer la percepción de los estudiantes sobre el programa de tutoría académica, en este se encontró que los aspectos personales ocupan el último lugar de los temas tratados en las sesiones de tutorías debido a que no todos los tutorados sienten confianza de manifestarle a su tutor situaciones de carácter privado aunado a ello los jóvenes presentan dificultad para integrarse al ambiente escolar y establecer relaciones interpersonales con sus compañeros y profesores, por lo cual el programa de tutorías como estrategia compensatoria durante el proceso de acompañamiento escolar estrategia central debe fortalecerse como vía combatir el rezago, la reprobación y el abandono escolar.

\section{Referencias bibliográficas}

Amaro, M., Méndez y Mendoza, F. (2014).Un estudio de las características profesionales del docente universitario para atender a la diversidad. Revista Latinoamericana de Educación Inclusiva, 8, 199216. Recuperado de repositoriocdpd.net:8080/handle/123456789/1702

Barcelata-Eguiarte, B., Gómez-Gutiérrez, Y. y Taboada, O. (2010), Perfil sociodemográfico del tutor. Revista Mexicana de Orientación Educativa, 7(19), 50-55. Recuperado de: http://pepsic.bvsalud.org/scielo.php? Script $=$ sci abstract\&pid $=$ S166575272010000200008\&lng=es\&nrm=iso\&tlng $=$ es 
Blanco, S. (s.f). La atención educativa a la diversidad: las escuelas inclusivas. En A, Marchesi, J,Telesco y C,Coll, Calidad, equidady reformas en la enseñanza (pp.7-169). España. Santillana.

Becete, F. y Domenéch, F. (1997). Motivación, aprendizaje y rendimiento escolar. Revista electrónica de motivación y emoción, 1, Recuperado de: http://reme.uji.es/articulos/pa0001/texto.html

Colmenares, A y Piñero, M. (2008). La investigación acción. Una herramienta metodológica heurística para la comprensión y transformación de realidades y prácticas socioeducativas. Revista de Educación, 14, 96-114. Recuperado de www.redalyc.org/articulo.oa?id $=7611189200$

Conejeros, M., Rojas, J y Segura, M. (2010) Confianza: un valor necesario y ausente en la educación chilena. Perfiles Educativos, 32, 30-46. Recuperado de http://www.scielo.org.mx/scielo.php?script=sci arttext\&pid=S0185-26982010000300003

Dopico, E. (2012). Tutoría universitaria: Propuestas didácticas de competencia tutorial. Revista de Docencia Universitaria, 11, 195-220. Recuperado de https://polipapers.upv.es/index.php/REDU/article/view/5573

DOF, (2016) Decreto por el que se organiza el Consejo Nacional de Fomento Educativo (CONAFE).

Ducoing, P. (2009). ¿'Tutoría y/o acompañamiento en educación? En Ducoing, P. Tutoría y mediación I. México: IISUE-UNAM

Gómez-Collado, M. (2012) La percepción de los estudiantes sobre el Programa de Tutoría Académica. Convergencia, Revista de Ciencias Sociales, 58, 209-233. Recuperado de: http://www.scielo.org.mx/pdf/conver/v19n58/v19n58a9.pdf

Gonzales, R. (2008). Herramientas básicas para el acompañamiento tutoral. Revista Mexicana de Orientación Educativa, 6, 12-18. Recuperado de http://pepsic.bvsalud.org/scielo.php?script=sci arttext\&pid=S1665-75272008000300003

Gordo, J y Serrano, A. (2008). Estrategias y prácticas cualitativas de la investigación social. México: Pearson.

García, A. y Adrogué, C. (2015). Abandono de los estudios universitarios: dimensión, factores asociados y desafíos para la política pública. Revista Fuentes, 16, 85-106, doi: http://dx.doi.org/10.12795/revistafuentes.2015.116.04

García, R., Cuevas, O., Vales, J y Cruz, I. (2012) Impacto del programa de tutorías en el desempeño académico de los estudiantes del Instituto Tecnológico de Sonora. Revista Electrónica de Investigación Educativa, 14, 106-121. Recuperado de http://redie.uabc.mx/vol14no1/contenido-garciaetal.html

Henández-Sampieri, R y Mendoza, C. (2018). Metodología de la investigación. Las rutas cuantitativa, cualitativa y mixta. México: Mc Graw Hill.

Landero, José Francisco Javier (2012). Deserción en la Educación Media Superior en México. Suma por la educación. La sociedad abriendo espacios a la calidad educativa. Recuperado http://www.sumaporlaeducacion. org.mx/ articulos-suma/desercion-de educacion-mediasuperior

Mckernan, J. (2001).Investigación-acción y currículum. España: Morata.

Moreno-Crespo, P. (2015). Educación a lo largo de la vida: aulas mayores. Revista Fuentes, 17, 113133, doi: http://dx.doi.org/10.12795/revistafuentes.2015.117.05.

Muñoz, B. (2004). La tutoría académica desde la perspectiva de la orientación educativa. Revista Mexicana de Orientación Educativa, 1, 1-7.

Luna, E., Gómez, J., Lasso, T (2012). Relación entre desempeño académico y autocontrol.

Ortiz, C., Mendoza, F y Méndez, J. (2016). Las miradas detrás de las cifras: el abandono escolar desde la experiencia de los jóvenes y sus profesores tutores. Revista Mexicana de Orientación Educativa 13, 44-51.

Osuna, C y Díaz, K. (2015) Estudio sobre jóvenes desertores de la educación media superior en Baja California, México. Congreso Nacional de Investigación Educativa. Chihuahua México.

Plá, S. (2018). Calidad educativa. Historia de una política para la desigualdad. México UNAM

Puerta Gil, C. A. (2016). El acompañamiento educativo como estrategia de cercanía impulsadora del aprendizaje del estudiante. Revista Virtual Universidad Católica del Norte, 49, 1-6. 
Robles, R. (2005). Orientación educativa y Rendimiento Académico. Revista Mexicana de Orientación Educativa, 4, 52-59.

Sánchez-Santamaría, J. y Manzanares, A. (2013). Tendencias internacionales sobre equidad educativa desde la perspectiva del cambio. Revista Electrónica de Investigación Educativa, 1, 12-28.

Sánchez-Santamaría, J. y Manzanares, A. (2017). Equidad y orientación en la educación secundaria. Madrid: La Muralla

Secretaria de Educación Pública [SEP]. Lineamientos de acción tutorial. Recuperado de http://www.dgb.sep.gob.mx/informacion-academica/actividadesparaescolares/acciontutorial/FI-LAT.pdf

Secretaria de Educación Pública [SEP]-Subsecretaria de Educación Media Superior [SEMS]. (2011). Siguele, caminemos juntos. Acompañamiento integral para jóvenes. Recuperado de https://www.dgb.sep.gob.mx/acciones-yprogramas/siguele/PROGRAMA_SIGUELE_DBASE.pdf

SEP. (2017). Ley General de Educación. Recuperado de: https://www.sep.gob.mx/work/models/sep1/Resource/558c2c24-0b12-4676-ad908ab78086b184/ley general_educacion.pdf

SEP. (2017). Lineamientos de acción tutorial. Recuperado de http://www.dgb.sep.gob.mx/informacionacademica/actividades-

SEP. (2018). Coordinación sectorial de desarrollo Académico. (2018). Recuperado de https://drive.google.com/drive/folders/1RFY9i3EQuvt27gxt87EjENXSUq_72oj3

Silva, M. (2015). La educación superior: énfasis renovados, repetidas estrategias. En La política educativa del sexenio 2013-2018. Alcances y limites (pp. 43-56). Recuperado de: http://www.ibero.mx/campus/publicaciones/La-politica-educativa-del-sexenio-20132018/pdf/La politica educativa.pdf.

Titscher, S., Meyer, M., Wodak, R. y Vetter, E. (2000). Methods of Text and Discourse Analysis. Londres: Sage.

\section{Para citar este artículo}

Boroel Cervantes, B.I., Sánchez Santamaría, J., Morales Gutiérrez, K.D., \& Henríquez Ritchie, P.S. (2018). Educación exitosa para todos: la tutoría como proceso de acompañamiento escolar desde la mirada de la equidad educativa. Revista Fuentes, 20(2), 91-104. [Fecha de consulta: dd $/ \mathrm{mm} / \mathrm{aa}]$.

doi: http://dx.doi.org/10.12795/revistafuentes.2017.v20.i2.06 